\title{
Chronic ethanol intake-induced changes in open-field behavior and cal- cium/calmodulin-dependent protein kinase IV expression in nucleus accumbens of rats: naloxone reversal ${ }^{1}$
}

\author{
Jing $\mathrm{LI}^{3}$, Wei-liang BIAN ${ }^{5}$, Gui-qin $\mathrm{XIE}^{4}$, Sheng-zhong $\mathrm{CUI}^{4}$, Mei-ling WU ${ }^{3}$, Yue-hua $\mathrm{LI}^{3}$, Ling-li QUE${ }^{3}$, Xiao-ru YUAN ${ }^{2,4}$ \\ Departments of ${ }^{3}$ Pathophysiology and ${ }^{4}$ Physiology, Nanjing Medical University, Nanjing 210029, China; ${ }^{5}$ Department of Infection, Nanjing \\ First Hospital, Nanjing 210006, China
}

\author{
Key words \\ ethanol; nucleus accumbens; calcium/ \\ calmodulin-dependent protein kinase IV; \\ open-field behavior; naloxone \\ ${ }^{1}$ This study was supported by the National \\ Natural Science Foundation of China (No \\ 30572173 and 30370449) \\ ${ }^{2}$ Correspondence to Prof Xiao-ru YUAN. \\ Phn 86-25-8686-3187. \\ Fax 86-25-8686-2888. \\ E-mailxryuan@public1.ptt.js.cn \\ Received 2007-12-20 \\ Accepted 2008-03-24 \\ doi: $10.1111 / \mathrm{j} .1745-7254.2008 .00805 . \mathrm{x}$
}

\begin{abstract}
Aim: To investigate the effects of chronic ethanol intake on the locomotor activity and the levels of calcium/calmodulin-dependent protein kinase IV (CaM kinase IV) in the nucleus accumbens (NAc) of rats. Simultaneously, the effects of nonselective opioid antagonist (naloxone) on the CaM kinase IV expression in the NAc and ethanol consumption of rats were also observed. Methods: Ethanol was administered in drinking water at the concentrations of $6 \%(v / v)$, for $28 \mathrm{~d}$. The locomotor activity of rats was investigated in the open-field apparatus. CaM kinase IV levels in the NAc were analyzed using Western blotting. Results: Rats consuming ethanol solution exhibited a significant decrease of ambulation activity, accompanied by a reduced frequency of explorative rearing in an open-field task on $\mathrm{d} 7$ and $\mathrm{d} 14$ of chronic ethanol ingestion, whereas presumed adaptation to the neurological effects of ethanol was observed on d 28. Chronic ethanol intake elicited a significant decrease of the CaM kinase IV expression in the nuclei, but not in the cytoplasm of the NAc on $\mathrm{d} 28$. Naloxone treatment significantly attenuated ethanol intake of rats and antagonized the decrease of CaM kinase IV in the nuclei of NAc neurons. The cytosolic CaM kinase IV protein levels of the NAc also increased in rats exposed to ethanol plus naloxone. Conclusion: Chronic ethanol intake-induced changes in explorative behavior is mediated at least partly by changes in CaM kinase IV signaling in the nuclei of the NAc, and naloxone attenuates ethanol consumption through antagonizing the downregulation of $\mathrm{CaM}$ kinase IV in the NAc.
\end{abstract}

\section{Introduction}

Like other addictive disorders, excessive consumption of alcohol (ethanol and ethyl alcohol) can lead to the addictive phenotypes of tolerance, dependence, and relapse after the cessation of drinking. The development of alcohol addiction involves the establishment of neuronal adaptation, which is a homeostatic response to ethanol-associated stimuli, and the formation of long-term synaptic plasticity occurring in the neural network ${ }^{[1]}$. Ethanol exerts both depressant and stimulant effects on locomotor activity in rodents. Low doses of ethanol increase the locomotor activity in mice while high doses decrease it. It has been reported that the repeated administration of ethanol elicits an adaptation to its behavioral effects ${ }^{[2]}$. This phenomenon has been implicated in the development of drug addiction or craving.

The family of multifunctional $\mathrm{Ca}^{2+}$ calmodulin-dependent protein serine/threonine kinases ( $\mathrm{CaM}$ kinase) includes $\mathrm{CaM}$ kinase I-IV and CaM kinase kinases ${ }^{[3,4]}$. Of the several isoforms of CaM kinase, CaM kinase IV is a calcium-dependent protein kinase that can be detected in both the nuclei and cytoplasm of neurons and is the only cAMP responsive-binding protein (CREB)-phosphorylating protein kinase that is detected predominately in the nuclei of neurons ${ }^{[5-7]}$. CaM kinase IV is associated with the protein serine/threo- 
nine phosphatase PP2A and has been thought to function as a regulatory module that controls CREB-mediated transcription in response to changes in nuclear $\mathrm{Ca}^{2+}$ concentration $^{[8]}$. This in turn leads to the activation of immediate early genes of the fos and jun families ${ }^{[9,10]}$ and neurotrophins, such as brain-derived neurotrophic factor ${ }^{[11]}$, which may regulate neuronal growth, differentiation of specific cells, and synaptic activity. Recent data from Ko et a $l^{[12]}$ suggest the important role of a nuclear protein kinase (CaM kinase IV) in both the behavioral and cellular adaptations following chronic morphine treatment.

The nucleus accumbens (NAc) in the central nerve system has been implicated in the reward and reinforcing properties of ethanol. Numerous studies implicate that CREB in the NAc plays an important role in cocaine reward mechanisms ${ }^{[13-15]}$. Previous data from our laboratory ${ }^{[16]}$ and other laboratories $^{[17,18]}$ also indicate that chronic ethanol intake downregulates the phosphorylation of CREB in the NAc. However, few have been linked CaM kinase IV signaling in the nuclei of NAc neurons to the development of behavioral adaptation following chronic ethanol treatment.

The endogenous opioid systems provide the brain with mechanisms that regulate not only opioid addiction, but the addiction of a variety of abused drugs. Many reports suggest that the endogenous opioid system as a relevant part of the neurobiological mechanisms is functionally involved in the reinforcement of drinking behavior and the development of alcohol dependence ${ }^{[19]}$. Therefore, the blockade of central opioid receptors using selective and non-selective opioid antagonists may modulate the positive reinforcing properties of ethanol and prove effective in reducing ethanol consumption $^{[20,21]}$. However, the mechanisms underlying their actions on ethanol intake remain unclear. In this study, we establish a link between CaM kinase IV and alcohol intake by examining the effects of non-selective opioid antagonist (naloxone) on the CaM kinase IV expression in the NAc and on ethanol intake in rats.

\section{Materials and methods}

Ethanol (code 040610660) was purchased from Nanjing Chemical Plant (Nanjing, China). Forty 1-month-old SpragueDawley rats, provided by the Experimental Animal Center of Jiangsu Province (Nanjing, China; certification № 97001), were housed in a quiet room with natural light and controlled temperature $\left(18-24^{\circ} \mathrm{C}\right)$. The animal experiments were in accordance with the guidelines for the care and use of animals provided by the Experimental Animal Center of Jiangsu Province.

Drug treatment Ethanol was administered in drinking water at a concentration of $6 \% v / v$ for $28 \mathrm{~d}$, according to Turchan et $a l^{[22]}$. Control animals received tap water for an identical period of time. For the naloxone studies, the ethanol-fed rats were injected intraperitoneally with naloxone (1. $0 \mathrm{mg} \cdot \mathrm{kg}^{-1} \cdot \mathrm{d}^{-1}$, purchased from Jingmei Biotech, Shanghai, China) or saline for $9 \mathrm{~d}$ from the 20th $\mathrm{d}$ of ethanol intake. Food and all ethanol solution or tap water were available ad libitum. Fresh water and ethanol solution were prepared and replaced at 7:00 every morning. There were 5 groups in the chronic ethanol and chronic naloxone studies: (1) intake of tap water for $28 \mathrm{~d}$ (control); (2) chronic intake of ethanol for $28 \mathrm{~d}$ (chronic ethanol); (3) withdrawal $24 \mathrm{~h}$ after $28 \mathrm{~d}$ of ethanol intake (withdrawal $24 \mathrm{~h}$ ); (4) ethanol intake plus chronic saline treatment (ethanol+vehicle); and (5) ethanol intake plus chronic naloxone treatment (ethanol+naloxone). There were 8 rats in each group. Ethanol consumption and the body weight of rats were monitored weekly.

Open-field activity The open-field test was carried out according to the method slightly modified from the works of Vassiljev et $a l^{[23]}$. The open-field apparatus used in the present investigation consisted of a square area $100 \mathrm{~cm} \times 50$ $\mathrm{cm}$ with $60 \mathrm{~cm}$-high walls. The floor was divided by lines into 8 equal squares. All measurements were obtained in a quiet room in the morning (10:00). To determine behavioral changes induced by chronic ethanol intake, rats from the control and chronic ethanol-intake groups were placed in the open field apparatus. The locomotor activity was respectively measured on $\mathrm{d} 7,14,21$, and 28 of ethanol treatment. The test was started by placing a rat in the center of area. Each test lasted for $5 \mathrm{~min}$, during which the number of squares crossed, and the number of rearings were recorded. Openfield behavior was evaluated by an observer unaware of the drug treatments.

Western blot analysis of CaM kinase IV protein levels Five experimental groups were included in this study: control, chronic ethanol, $24 \mathrm{~h}$ ethanol withdrawal, ethanol+vehicle, and ethanol+naloxone. Rats from the 5 groups were anesthetized and the brains were immediately removed. The NAc were dissected bilaterally and homogenized on ice. Nuclear and cytosolic fractions were obtained with a nuclear extract kit (Active Motif), and protein contents were determined by the Pierce protein assay reagent (Pierce). Samples of protein $(60 \mathrm{mg})$ were diluted in sample buffer and boiled for $5 \mathrm{~min}$. Samples were subjected to SDS-PAGE (100 V for $2 \mathrm{~h}$ ) and then transferred electrophoretically to nitrocellulose membranes. The membranes were rinsed and incubated in blocking solution for $1 \mathrm{~h}$ at room temperature. The anti-CaM kinase IV polyclonal antibody $(1: 2000$, BD Transduction Laboratories) was incubated overnight at $4{ }^{\circ} \mathrm{C}$. After washing, 
the blots were incubated for $1 \mathrm{~h}$ at room temperature with peroxidase-labeled goat antimouse immunoglobulin $\mathrm{G}$ (1: 2000). The detection of labeled proteins was accomplished with diaminobenzidine. The optical densities of the CaM kinase IV bands were analyzed using ImageJ software (NIH).

Statistical analysis The two-tailed Student's $t$-test was applied for statistical evaluation. Data were expressed as mean \pm SD. The statistical criterion for significance was $P<0.05$.

\section{Results}

Acquisition of ethanol and body weight during chronic ethanol exposure The daily average ethanol consumption of ethanol-treated rats was $6.52 \pm 0.38 \mathrm{~g}$ of absolute ethanol $/ \mathrm{kg}$ body weight. There were no obvious changes in the acquisition of ethanol intake during the entire experimental period (Table 1). Control and chronic ethanol rats were weighed once a week to observe the effect of ethanol on the rat body weight. At the beginning of the experiment, there were no differences in body weight between the control and ethanolfed rats. Ethanol consumption did not generally differ in the body weight gain between the ethanol-fed rats (352.7 $\pm 26.6 \mathrm{~g})$ and the control rats $(358.3 \pm 25.2 \mathrm{~g}$; Table 1$)$.

Behavioral changes The behavioral activities of the ethanol-treated and control rats in response to a novel environment were compared in the open-field on $\mathrm{d} 7,14,21$, or 28 of chronic ethanol ingestion. Chronic ethanol intake resulted in a dramatically decreased horizontal locomotor activity (ambulation) by a maximum of $64 \%$ of the control value on $\mathrm{d}$ 7 of ethanol treatment $(P<0.01$; Figure 1A); this was maintained up to $21 \mathrm{~d}$ of ethanol treatment, whereas the difference in line crossings between the ethanol-fed and control groups disappeared on $\mathrm{d} 28$ of chronic ethanol intoxication [23.7 \pm 7.5 (ethanol) vs 22.3 \pm 5.9 (control), ambulation score; Figure 1A]. In parallel with the changes in the horizontal locomotor movements, similar effects of chronic ethanol treatment on the frequency of rearing activity were recorded (Figure 1B). The suppressant effect of chronic ethanol ingestion on exploratory behavior was most apparent on $\mathrm{d} 7$ and 14 of ethanol treatment $(P<0.01, P<0.05$; Figure 1B). Interestingly, the difference in the frequency of rearing activity between the ethanol-fed and control groups disappeared on $\mathrm{d} 21$ of the ethanol treatment. There was also no difference on $\mathrm{d} 28$.

Changes of CaM kinase IV protein levels in the NAc of rats Western blots were used to determine the changes of CaM kinase IV in the NAc of rats during chronic ethanol exposure. Figure 2A shows representative Western blots of $\mathrm{CaM}$ kinase IV in the cytoplasm and nuclei of NAc neurons. Ethanol given to rats in drinking water led to a significant reduction, compared to the controls, in the expression of CaM kinase IV protein in the nuclei of NAc neurons (Figure 2C), whereas the suppressant effect disappeared at $24 \mathrm{~h}$ after ethanol withdrawal. There were no changes of CaM kinase IV protein levels in the cytoplasm of NAc neurons (Figure 2A). These results indicated that the decrease of CaM kinase IV protein induced by chronic ethanol exposure occurred only in the nuclei of NAc neurons.

Effects of naloxone treatment on the acquisition of ethanol intake in rats After the rats were given 6\% ethanol for $20 \mathrm{~d}$, they began to receive a naloxone or vehicle (saline) injection daily for $9 \mathrm{~d}$. The pattern of drinking behaviors from 4-9 $d$ with or without naloxone treatment is shown in Figure 3. The rats treated with naloxone consumed significantly less ethanol solution on the 4th day of treatment, compared with the rats treated with vehicle. It was maintained until the end of the experiment.

Effects of naloxone treatment on CaM kinase IV expression in the NAc of rats Chronic naloxone treatment could antagonize the decrease of CaM kinase IV expression in the nuclei of NAc neurons (Figure 2B). The levels of CaM kinase IV protein in the nuclei of NAc of rats fed with ethanol

Table 1. Changes of rat body weight and ethanol consumption in control and ethanol-fed groups during chronic ethanol intake. Values are mean $\pm \mathrm{SD}$ from 8 rats at each time point.

\begin{tabular}{|c|c|c|c|c|c|c|}
\hline & \multicolumn{6}{|c|}{ Day of ethanol treatment } \\
\hline & & 1 & 7 & 14 & 21 & 28 \\
\hline Body & Control & $142.00 \pm 8.92$ & $202.17 \pm 22.99$ & $260.97 \pm 19.83$ & $326.00 \pm 24.44$ & $358.33 \pm 25.24$ \\
\hline weight (g) & Ethanol & $142.31 \pm 7.72$ & $193.44 \pm 18.60$ & $243.41 \pm 21.09$ & $299.29 \pm 29.62$ & $352.65 \pm 26.60$ \\
\hline $\begin{array}{l}\text { Ethanol consumption } \\
\text { ( } \mathrm{g} / \mathrm{kg} \text { body weight) }\end{array}$ & Ethanol & $6.01 \pm 0.60$ & $6.90 \pm 0.55$ & $6.88 \pm 1.05$ & $6.49 \pm 0.76$ & $6.34 \pm 0.65$ \\
\hline
\end{tabular}

Ethanol consumption and body weight of rats were monitored weekly. There were no significant differences in body weight between control and chronic ethanol rats during the entire experiment. Average of daily ethanol consumption was $6.52 \pm 0.38 \mathrm{~g}$ of absolute ethanol/kg body weight. 
$\mathbf{A}$
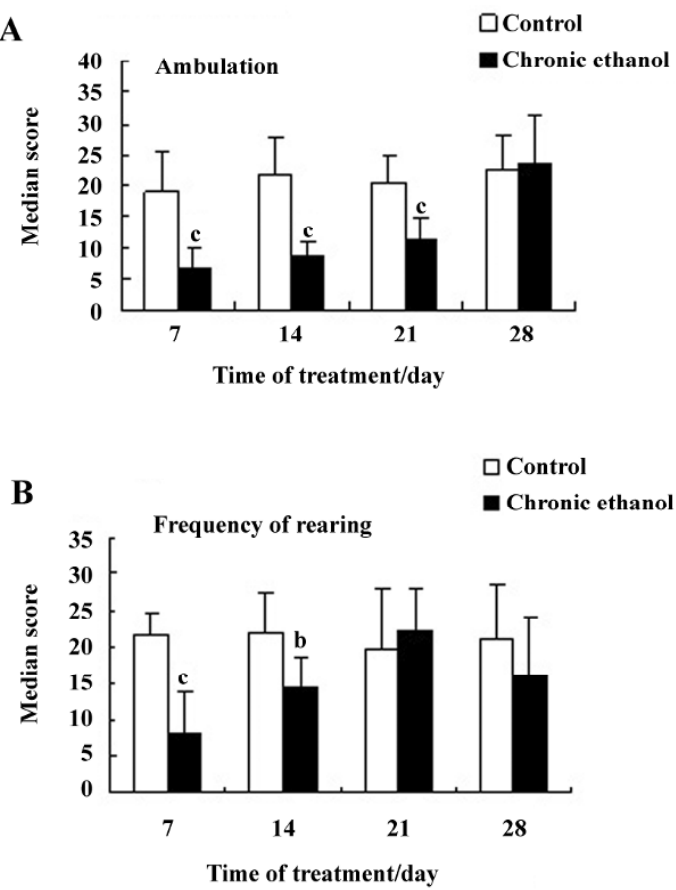

Figure 1. Intensity of horizontal locomotion (ambulation, A) and rearing of control and ethanol-fed rats in the open-field apparatus after $7,14,21$, or $28 \mathrm{~d}$ of chronic ethanol exposure. Eight control and ethanol-treated rats were used in each time point to determine behavioral activities in the open-field apparatus. ${ }^{\mathrm{b}} P<0.05,{ }^{\mathrm{c}} P<0.01$ for ambulation and frequency of rearing, respectively, $v s$ control rats. Data are reported as mean $\pm \mathrm{SD}$. plus naloxone were higher $(P<0.01)$ than the rats fed with ethanol plus vehicle (Figure 2D). Additionally, naloxone treatment also slightly increased the expression of $\mathrm{CaM}$ kinase IV protein in the cytoplasm of NAc neurons $(P<0.05$; Figure 2D).

\section{Discussion}

In the present study, we demonstrated that chronic ethanol intake resulted in a dramatic decrease of explorative behaviors after 7 and 14 days of ethanol treatment, whereas the presumed adaptation to the neurological effects of ethanol was observed on $\mathrm{d} 28$. Chronic ethanol ingestion also led to a significant reduction of CaM kinase IV levels in the nuclei of the NAc. Naloxone treatment attenuated ethanol consumption of rats and antagonized the decrease of CaM kinase IV in the nuclei of NAc neurons. Our data suggested that changes in explorative behavior induced by chronic ethanol intake may involve the downregulation of CaM kinase IV in the nuclei of NAc. Naloxone attenuates ethanol intake through antagonizing the downregulation of CaM kinase IV in the NAc.

In the present investigation, the drinking water of rats was replaced with a $6 \%(v / v)$ ethanol solution in the water, and with free access to standard rodent diet. At the end of the experiment, no significant differences in body weight
A
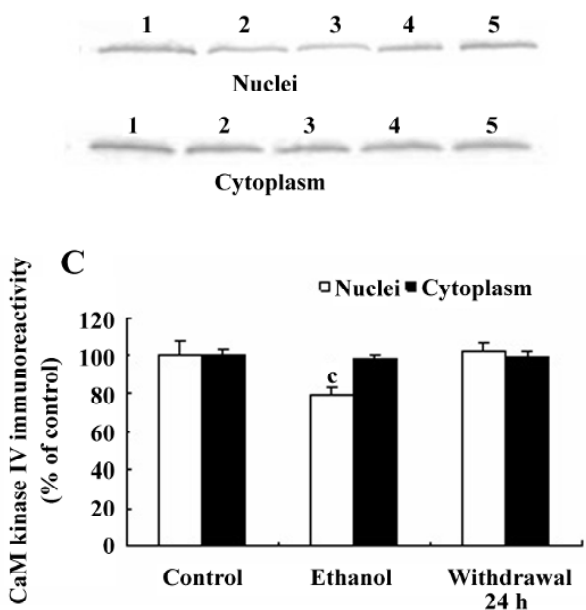

B

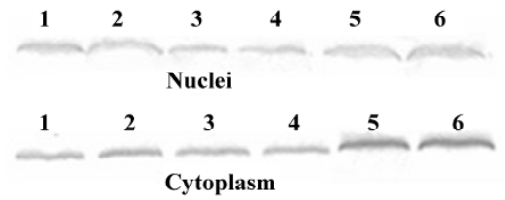

D

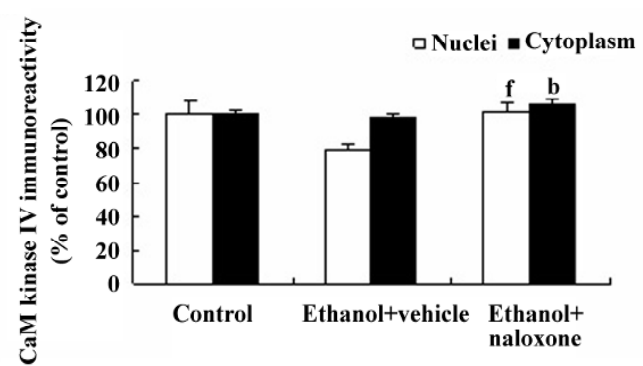

Figure 2. (A) representative Western blots of CaM kinase IV in the nuclei and cytoplasm of the NAc of control, chronic ethanol intake, and ethanol-withdrawn rats. Lane 1, control; lanes 2 and 3, chronic ethanol; lanes 4 and 5, ethanol withdrawal for $24 \mathrm{~h}$. (B) representative Western blots of CaM kinase IV in the nuclei and cytoplasm of the NAc of control, ethanol+vehicle, and ethanol+naloxone-treated rats. Lanes 1 and 2, control; lanes 3 and 4, chronic ethanol intake+saline treatment; lanes 5 and 6, chronic ethanol intake + naloxone treatment. (C) effects of chronic ethanol exposure on CaM kinase IV protein levels in the nuclei and cytoplasm of the rat NAc neurons. Data are mean \pm SD of 8 rats and are represented as a percentage of the control. (D) Naloxone treatment increases the levels of CaM kinase IV protein in the NAc of rats exposed to ethanol. ${ }^{\mathrm{b}} P<0.05,{ }^{\mathrm{c}} P<0.01 \mathrm{vs}$ control rats. ${ }^{\mathrm{f}} P<0.01 \mathrm{vs}$ ethanol-fed rats with vehicle. 


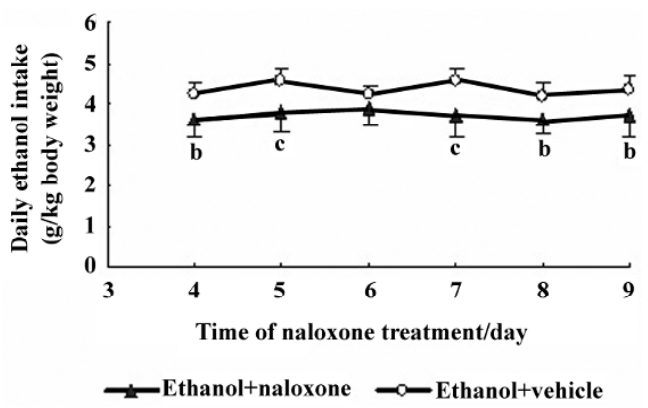

Figure 3. Effects of naloxone treatment on ethanol consumption of rats. Naloxone or saline were administrated to rats exposed to ethanol for $20 \mathrm{~d}$. Values are mean $\pm \mathrm{SD}$ from 8 rats at each time point. ${ }^{\mathrm{b}} P<0.05,{ }^{\mathrm{c}} P<0.01$ vs ethanol-fed rats with vehicle.

and acquisition of ethanol intake were observed between the control and ethanol-exposed rats, which may suggest that continuous ethanol intake does not affect the food and ethanol intake of rats. Using a similar procedure of ethanol administration, obvious changes in the blood ethanol level were not found during the entire chronic ethanol treatment period $^{[24]}$. Therefore, it can be thought that alterations of behavior and protein expression in ethanol-exposed rats were only associated with the stimulation of repeated ethanol intake, not because of insufficient food or ethanol accumulation in blood.

The administration of ethanol has been shown to modulate locomotor activity. In the present investigation, we found that chronic ethanol exposure (for $28 \mathrm{~d}$ ) exerted profound effects on the novelty-induced explorative behavior. The horizontal motor activity and the frequency of rearing were significantly decreased in the animals subjected to chronic ethanol intoxication. It was worth noting that the differences in ambulation and rearing activity were reduced after a $28 \mathrm{~d}$ treatment period, which may reflect the adaptation to and compensation of the direct effects of ethanol. In our laboratory, we have demonstrated ${ }^{[24]}$ that chronic ethanol exposure suppresses the induction of corticostriatal longterm depression, and the suppressive effects weakened time dependently during the period between day 20 and 30, which also indicates that neuroadaptations occurred in the striatum after chronic ethanol intake. These adaptive alterations in corticostriatal synaptic plasticity in rats chronically exposed to ethanol are highly congruent with changes of behaviors mentioned earlier, which may be associated with the development of tolerance after long-term alcohol drinking. However, the molecular mechanisms of these adaptations in behavior and synaptic plasticity remain unclear.

A number of studies have implicated CaM kinase IV in the control of gene transcription by regulating the activity of a number of different transcription factors ${ }^{[9,25]}$. Among them, CREB is the only known direct substrate for the enzyme. Previous data have indicated that chronic ethanol intake could downregulate the phosphorylation of CREB in the nuclei of NAc neurons ${ }^{[16]}$. In present investigation, we found that a significant decrease in the expression of CaM kinase IV protein induced by chronic ethanol exposure occurred mainly in the nuclei of NAc neurons, simultaneously accompanied by adaptative alterations in locomotor activity. Misra et $a l^{[17]}$ also reported that chronic ethanol intake significantly decreased the expression of CaM kinase IV, especially in the shell of the NAc. From the present data and our previous investigations of the decreased CREB phosphorylation in the nuclei of NAc neurons with similar experimental protocols $^{[16]}$, we postulate that the decrease of CaM kinase IV protein in the nuclei of NAc neurons may be responsible for the downregulation of CREB phospho-rylation, which then contributes to the adaptations that occur in behaviors following chronic ethanol exposure. The present investigation does not address the cause and effect relationship between CaM kinase IV signaling in NAc and behavioral adaptations, but rather provides evidence to suggest that CaM kinase IV signaling in the nuclei of NAc neurons is sensitive to chronic alcohol drinking and is most likely involved in ethanol-dependent mechanisms. Future studies will elucidate the precise nature of the involvement of CaM kinase IV in the dependent properties of ethanol.

Another observation of the present study is that opioid antagonist (naloxone) treatment is able to attenuate alcohol consumption in rats. This is also associated with antagonism of ethanol-induced reductions in CaM kinase IV expression in the nuclei of NAc neurons. It is possible that lower CaM kinase IV signaling may represent the neuroadaptation in NAc due to which rats would like to continue drinking to increase the CaM kinase IV function. However, the mechanism by which naloxone antagonizes the effects of ethanol on CaM kinase IV signaling is not clear. It has been shown that naloxone could increase the levels of cytoplasmic $\mathrm{Ca}^{2+}$ in smooth muscle cells pre-incubated with morphine which may be associated with the phosphorylation of CREB enhancement ${ }^{[26]}$. Recent studies reported that CaM kinase IV expression was strongly localized with the $\mu$-opioid receptor after chronic morphine treatment in many brain areas ${ }^{[27]}$. This colocalization suggests that CaM kinase IV may be an important member in a pathway connecting the activation of the $\mu$-opioid receptor to CREB. Previous studies from our laboratory have indicated that long-term naloxone treatment can antagonize the downregulation of CREB phosphorylation in the NAc in- 
duced by chronic ethanol exposure ${ }^{[16]}$, which suggests that the inhibitory actions of ethanol on the phosphorylation of CREB in NAc may be involved in the activation of the opiate receptor. In present study, we found that the administration of naloxone to chronic ethanol-fed rats exerted a significant increase of CaM kinase IV expression not only in the nuclei, but also in cytoplasm of NAc neurons. Therefore, our studies may provide evidence that the blockade of the downregulation of CaM kinase IV by naloxone may increase CREB phosphorylation in the NAc during chronic ethanol intake and then reduce ethanol consumption.

In conclusion, our results show that chronic ethanol ingestion $(28 \mathrm{~d})$ results in a correlative adaptation of explorative behavioral function and the decrease of CaM kinase IV protein expression in the nuclei of NAc neurons in rats. The present findings may provide evidence to suggest that plasticity-associated neural adaptation within the neurocircuitry of NAc during chronic ethanol intake may depend upon the decreased CaM kinase IV-related gene transcription factor. Furthermore, these molecular adaptations in the NAc may cause habit formation towards alcohol drinking behaviors that ultimately lead to the development of alcohol addiction.

\section{Acknowledgements}

We thank the Innovation Center for Medical Graduate Studies Jiangsu Province for their pertinent and substantial advice for this study.

\section{Author contributions}

Xiao-ru YUAN and Jing LI designed research; Jing LI, Wei-liang BIAN, Gui-qin XIE, Sheng-zhong CUI, and LingLi QUE performed research; Yue-hua LI, Sheng-zhong CUI and Ling-li QUE contributed new reagents or analytic tools; Jing LI, Wei-liang BIAN, Mei-ling WU, and Gui-qin XIE analyzed data; Xiao-ru YUAN, Jing LI and Mei-ling WU wrote the paper.

\section{References}

1 Berke JD, Hyman SE. Addiction, dopamine, and the molecular mechanisms of memory (review). Neuron 2000; 25: 515-32.

2 Harkany T, Sasvari M, Nyakas C. Chronic ethanol ingestioninduced changes in open-field behavior and oxidative stress in the rat. Pharmacol Biochem Behav 1997; 58: 195-201.

3 Schulman H, Braun A. Calcium/calmodulin-dependent protein kinase. In: Carafoli E, Klee C, editors. Calcium as a cellular regulator. New York: OUP; 1999. p 311-43.

4 Means AR. Regulatory cascades involving calmodulin-dependent protein kinases. Mol Endocrinol 2000; 14: 4-13.

5 Jensen KF, OhmstedeCA, Fisher RS, Sahyoun N. Nuclear and axonal localization of $\mathrm{Ca}^{2+} /$ calmodulin-dependent protein kinase type Gr in rat cerebellar cortex. Proc Natl Acad Sci USA 1991; 88: $2850-3$.

6 Nakamura Y, Okuno S, Sato F, Fujisawa H. An immunohistochemical study of $\mathrm{Ca}^{2+} /$ calmodulin-dependent protein kinase IV in the rat central nervous system: light and electron microscopic observations. Neuroscience 1995; 68: 181-94.

7 Kang H, Sun LD, Atkins CM, Soderling TR, Wilson MA, Tonegawa S. An important role of neural activity-dependent CaMKIV signaling in the consolidation of long-term memory. Cell 2001; 106: 771-83.

8 Westphal RS, Anderson KA, Means AR, Wadzinski BE. A signaling complex of $\mathrm{Ca}^{2+} /$ calmodulin-dependent protein kinase IV and protein phosphatase 2A. Science 1998; 280: 1258-61.

9 Bito H, Deisseroth K, Tsient RW. CREB phosphorylation and dephosphorylation: a $\mathrm{Ca}^{2+}$ - and stimulus duration-dependent switch for hippocampal gene expression. Cell 1996; 87: 1203-14.

10 Chawla S, Hardingham GE, Quinn DR, Bading H. CBP: a signalregulated transcriptional coactivation controlled by nuclear calcium and CaM kinase IV. Science 1998; 281: 1505-9.

11 Shieh PB, Hu SC, Bobb K, Timmusk T, Ghosh A. Identification of a signaling pathway involved in calcium regulation of BDNF. Neuron 1998; 20: 727-40.

12 Ko SW, Jia Y, Xu H, Yim SJ, Jang HD, Lee YS, et al. Evidence for a role of CaMKIV in the development of opioid analgesic tolerance. J Neurosci 2006; 23: 2158-68.

13 Carlezon WA Jr, Thome J, Olson VG, Lane-Ladd SB, Brodkin ES, Hiroi N, et al. Regulation of cocaine reward by CREB. Science 1998; 282: 2272-5.

14 Self DW, Genova LM, Hope BT, Barnhart WJ, Spencer JJ, Nestler EJ. Involvement of cAMP-dependent protein kinase in the nucleus accumbens in cocaine self-administration and relapse of cocaine-seeking behavior. J Neurosci 1998; 18: 1848-59.

15 McClung CA, Nestler EJ. Regulation of gene expression and cocaine reward by CREB and delta FosB. Nat Neurosci 2003; 6: 1208-15.

16 Li J, Li YH, Yuan XR. Changes of phosphorylation of cAMP response element binding protein in rat nucleus accumbens after chronic ethanol intake: naloxone reversal. Acta Pharmacol Sin 2003; 24: 930-6.

17 Misra K, Roy A, and Pandey SC. Effects of voluntary ethanol intake on the expression of $\mathrm{Ca}^{2+} /$ calmodulin-dependent protein kinase IV and on CREB expression and phosphorylation in the rat nucleus accumbens. Neuroreport 2001; 12: 4133-7.

18 Pandey SC, Roy A, Zhang H, Xu T. Partial deletion of the cAMP response element-binding protein gene promotes alcohol-drinking behaviors. J Neurosci 2004; 24: 5022-30.

19 Roozen HG, de Waart R, van der Windt DA, van den Brink W, de Jong CA, Kerkhof AJ. A systematic review of the effectiveness of naltrexone in the maintenance treatment of opioid and alcohol dependence. Eur Neuropsychopharmacol 2006; 16: 311-23.

20 Fachin-Scheit DJ, Frozino RA, Pigatto G, Oliveira Goeldner F, Boerngen de Lacerda R. Development of a mouse model of ethanol addiction: naltrexone efficacy in reducing consumption but not cravings. J Neural Transm 2006; 113: 1305-21.

21 Camarini R, Pires MLN, Calil HM. Involvement of the opioid system in the development and expression of sensitization to the locomotor-activating effect of ethanol. Int J Neuropsychopharmacol 2000; 3: 303-9. 
22 Turchan J, Przewlocka B, Toth G, Lason W, Borsodi A, Przewlocki $\mathrm{R}$. The effect of repeated administration of morphine, cocaine and ethanol on $\mathrm{mu}$ and delta opioid receptor density in the nucleus accumbens and striatum of the rat. Neuroscience 1999; 91: 971-7.

23 Vassiljev V, Mesila I, Vali M, Pokk P. The influence of the nitric oxide synthase inhibitor L-NOARG on the effects of ethanol in rats after acute ethanol administration. Pharmacol Toxicol 2000; 86: $63-7$

24 Xia JX, Li J, Zhou R, Zhang XH, Ge YB, Yuan XR. Alterations of rat corticostriatal synaptic plasticity after chronic ethanol exposure and withdrawal. Alcohol Clin Exp Res 2006; 30: 819-24.
25 Tardito D, Tiraboschi E, Kasahara J, Racagni G, Popoli M. Reduced CREB phosphorylation after chronic lithium treatment is associated with down-regulation of CaM kinase IV in rat hippocampus. Int J Neuropsychopharmacol 2007; 10: 491-6.

26 Takeba Y, Suzuki N, Kaneke A, Asai T, Sakane T. Endorphin and enkephalin ameliorate excessive synovial cell functions in patients with rheumatoid arthritis. J Rheumatol 2001; 28: 2176-83.

27 Nemmani KV, Lalonde J, Mogil JS. Region-specific changes of calcium/calmodulin-dependent protein kinase IV in the mouse brain following chronic morphine treatment. Neuroreport 2005; 16: 879-82.

\title{
The 7th Chinese Symposium on Ca Signaling (CSCS)
}

\author{
Yichang, Hubei province
}

July 22-24, 2008

Please contact with Dr Shun-yi WEI, wsy@moon.ibp.ac.cn. 